كلمة التحرير

\title{
الإسلامية في "إسلامية المعرفة"
}

مصطلح إسلامية المعرفة، بهذه الصيغة، مصطلح حديث يحاول الربط بين المعرفة بميادينها المختلفة، والإسلام من حيث كونه رؤية توحيدية كونية.

وأصبح المصطلح شعاراً لجملة من النشاطات الفكرية، وعَلَماً على ابتحٍٍ فكري له نظةٌٌ خاصة لمجمل قضايا الأمة والعالم فالإسلامية هي نسبة إلى الإسلام وانتساب إليه، وهو مصطلح يقابل مصطلحات أخرى تتعلق بواقع الأمة ومشاريع غوضها. ومن المصطلحات المقابلة للإسلامية: القومية، والتحديث، والتغريب، والعلمانية.

و "الإسلامية" مصطلح أبو الحسن الأشعري في القرن الرابع الهجري، الذي ميز بوساطته "مقالات الإسلاميين"، الذين يجمعهم الإسلام ويشتمل عليهم رغم تشتتهم وتفرقهم جماعات وأحزاباً، عن "مقالات غير الإسلاميين" من الملحدين واستخدام الكواكبي في القرن الماضي نفس المصطلح الإسلامي، ليعني به نظام الحكم الذي يطبقه المسلمون في حياتم، واقترح الإسلامية شعاراً يتضمن مجموعة من المبادئ هي المساواة، والعدالة، والحرية، والشورى الدستورية، وذلك لمواجهة الاستبداد الذي كان يسود أركان الدولة والأمة، وحاول بهذا التمييز أن يقدم شعاراً يحفز على النهوض والحركة، بدلاً من الإسلام الذي أصبح عَلَماً على المعنى الرسمي للدين في عصره.

وقد ورد استخدام المصطلح في مطلع السبعينيات في خطاب اتحادات الطلبة المسلمين في الغرب وبمعية الاجتماعيين المسلمين في الولايات المتحدة ليؤكد على الحاجة إلى البدء في "عملية" الأسلمة Islamization المطلوبة للمعرفة في العلوم الاجتماعية على وجه الخصوص، من أجل تحقيق النهوض الحضاري للأمة الإسلامية. ثم شاع استخدام مصطلح "الأسلمة" في الخطاب التحريضي الإيديولوجي للدعاة والحركيين الإسلاميين طيلة السبعينات وما بعدها، من أجل المطالبة بأسلمة التشريعات والقوانين وتطبيق الشريعة الإسلامية، وأصبح من المفردات المستعملة في خطاب الصحوة الإسلامية وفي تقارير المراقبين والباحثين الغربيين المتخصصين في رصد الصحوة وتطوراتا. 
وفي حين لقي مصطلح الأسلمة رواجاً في كثير من البلدان الإسلامية غير العربية فإنه واجه اعتراضات أساسية في عدد من البلدان العربية سواء لدى المثقفين غير الإسلاميين الذين لا يقبلون أن يجدوا أنفسهم خارج محيطهم الإسلامي أو لدى الحكام الذين لا يقبلون أن يوصفوا بالكفر والجاهلية إذا واجهوا تيار الأسلمة. واستدعت هذه التحفظات استخدام مصطلحات أقل حرجاً مثل "التأصيل الإسلامي" و "التوجيه الإسلامي" و "الصياغة الإسلامية" وإعمال "المنظور الإسلامي" وغيرها.

وإذا كان مصطلح "الإسلامية" قد وجد اعتراضات لدى غير الإسلاميين من المثقفين والمفكرين العلمانيين، باعتباره - في نظرهم- محاولة لسحب بساط الشرعية السياسية والاجتماعية التي تمتعوا بها في العهود السابقة، فقد كان النكير على مصطلح "إسلامية المعرفة" أكثر شدة، وبخاصة من أولئك المفكرين العلمانيين الذين يعملون في القطاع الأكاديمي، الذي طالما تندروا بوصف المعرفة بالإسلام أو بأي دين غيره، باعتبار أن المعرفة هي نتاج نشاط إنساني عالمي وأن النتاج موضوعي وحيادي لا دين له. وكثير من الكتابات المعارضة لفكرة الإسلامية، سواءً من داخل الدائرة الإسلامية أو من خارجها، هو استخفاف لا يستحق الاهتمام، ولكن بعض هذه الكتابات حاول أن ينحو منحى علمياً معرفياً رصيناً. يستحق معه شيئاً من الاهتمام والمناقشة، قد لا يكون مجاها هذا التقديم.

ومع ذلك فإنه يجب الانتباه إلى البعد السلبي الذي يمكن لوصف "الإسلامي" أن يوحي به في ظل تخلف المسلمين، وشعورهم بالنقص، والصور النمطية المتحيزة في أذهان الآخرين عنهم. وقد يظهر هذا البعد السلبي في تصور ظلال الحصرية والخصوصية، التي تقصر صلاحية المفاهيم والمبادئ "الإسلامية" وتعاملها على إتباع دين معين، أو بناء أمة معينة، في حين أن الإسلام دين عالمي جاء للناس كافة، وأنه صالح لكل زمان ومكان، وأنه رحمة للعالمين. ويتوقع أن تزول الحاجة إلى استخدام لفظ "الإسلامي" مع توسع دائرة الممارسات الإسلامية في مجتمعات المسلمين، وتنتفي بذلك مبررات حصر الخصائص الإسلامية، وقصرها على ممارسة معينة، أو جامعة، أو مستشفى، أو جمعية، أو حزب، عندما تصطبغ جميع المؤسسات بالصبغة الإسلامية.

وكذلك فإن وصف "الإسلامي" للنظام الاقتصادي، أو التربوي، أو المعرفي، سوف يفقد مبرره في داخل الدائرة الحضارية الإسلامية؛ لأن التطور والنمو الفكري سوف يتجاوز عموميات الانتماء العام ليدخل في تفاصيل هذه الأنظمة، وعندها تتعدد الاجتهادات والنظريات في تفاصيل النظام التربوي مثلاً، مع صحة انتساجها جميعاً إلى 
الإسلام. وقد تظهر الحاجة عندها إلى نسبة كل نظرية إلى اسم العالم المسلم الذي يطورها، وقد تنسب المؤسسات التعليمية التي تنشأ لتطبيق نظرية معينة في التعليم إلى اسم العالم المسلم الذي يطور تلك النظرية، أو اسم الحاكم الذي

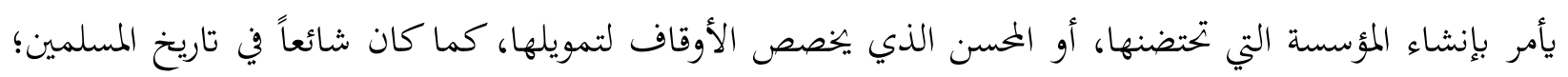
مثل المدارس النظامية نسبة إلى نظام الملك (485هـ) وزير السلطان السلجوقي، وكما هو شائع في مجتمعات اليوم مثل مدارس مونتيسوري، نسبة إلى الطبية والمربية الإيطالية ماريا مونتيسري (1952-1870م).

والإسلامية في "إسلامية المعرفة" من الناحية اللغوية مصدر صناعي للإسلام، ويختلف المصدر في معناه عن الاسم المنسوب الناتج عن إضافة ياء النسبة وتاء التأنيث في أن المراد أمر آخر غير وصف المعرفة بأها إسلامية، حين نقول: إن المعرفة الإسلامية تطورت مع تطور المجتمع الإسلامي؛ أي المعرفة التي أبدعتها عقول الباحثين والعلماء في الججتمع الإسلامي ضمن النظام المعرفي الإسلامي والدائرة الحضارية الإسلامية، أو حين نقول: إن المعرفة الإسلامية تعتمد الوحي والكون مصدرين أساسيين، بينما تعتمد المعرفة الغربية المعاصرة الكون فقط وتستبعد الوحي أن يكون له أية مرجعية.

أما صيغة المصدر الصناعي في "إسلامية " المعرفة فإنه يفيد في المقابل مجموعة من الصفات الخاصة باللفظ ويكون المستفاد بهذا المصدر الصناعي كالمستفاد بالمصدر في دلالثه المعنوية، حين نقول: تتبنى هذه المجلة إسلامية المعرفة كما نقول تؤكد المجلة ضرورة انتساب المعرفة إلى الإسلام وصدورها عن نظامه الاعتقادي وصياغتها وتوظيفها ضمن نظامه القيمي، بمعنى تدعو المجلة أن يكون منهج المعرفة إسلامياً. وقد يشتبه المنسوب بالمصدر الصناعي في مثل قولنا: تتصف المعرفة التي تتبناها المجلة بالإسلامية بمعنى المنهجية الإسلامية أو الاتحاه الإسلامي.

ويشبه المصدر الصناعي أن يكون صيغة مستحدثة، دعت إليها دواعي التوسع في مواجهة حاجات التعبير وقد أكثر منه المولودون عند استخدام المصطلحات وبخاصة في أثناء الترجمة إلى العربية وأصبح متعارفاً أن يستفاد بالمصدر الصناعي في مثل إسلامية المعرفة: أي كون المعرفة إسلامية، للتميز اللطيف بين المراد بالمنسوب وبالمصدر. وقد نجم عن ذلك فرق آخر في الصياغة النحوية لكلٍ منهما، إذ يقع المنسوب وصفاً في الغالب: معرفة إسلامية بينما يقع المصدر الصناعي مضافاً في الغالب: إسلامية المعرفة. 
وعندما كانت الأمة الإسلامية في أوج عطائها وازدهار حضارتها، لم يكن علماء الأمة يستخدمون مصطلح "الإسلامي" في مؤلفاقم في غختلف العلوم وفي مؤسسات المجتمع. فاكتفى الإمام الشافعي (204هـ) باسم "الرسالة " لكتابه في أصول الفقه؛ والإمام مالك (197هـ) اكتفى باسم "الموطأ" لكتابه في الحديث؛ والقاضي أبو يوسف (182هـ) اكتفى باسم "الخراج" لكتابه في الاقتصاد؛ وابن سينا (428هـ) اكتفى باسم "القانون" لكتابه في الطب و"النجاة" لكتابه في الفلسفة، والحسن بن الهيثم (430هـ) اكتفى باسم "المناظر" لكتابه في الفيزياء؛ وإمام الحرمين الجويني (478هـ) اكتفى باسم "الورقات" لكتابه في الأصول، وهكذا.

ثم تدهورت أوضاع المجتمع الإسلامي، وفقد خصائصه الإسلامية وغابت عنه الممارسات الإسلامية في شؤون الحياة المختلفة، وطغى الفكر الغربي العلماني وسادت مفاهيمه ومصطلحاته، ولاسيما في فترة الاستعمار والتبعية له بعد الاستقلال؛ فوجد العلماء والدعاة إلى استئناف الحياة الإسلامية، في مجتمعات المسلمين، مبرراً لإضافة اسم "الإسلامي" و "الإسلامية" إلى المفاهيم والمصطلحات والجماعات والمؤسسات، التي يتم تطويرها في بجالات العطاء الفكري الإسلامي، وفي مجالات تطور النماذج الإسلامية البديلة. وخدمت هذه الصفة الإسلامية، كما يبدو في تأكيد الهوية والانتساب إلى دائرة الحضارة الإسلامية المختلفة عن معطيات الدائرة الحضارية الغربية السائدة، ولعلها خدمت أيضاً في بيان دور الرسالة التي يمكن لهذا العطاء الفكري المتميز أن يقدمها إلى الآخرين، ويسهم من خلالها في تأكيد فاعليته في التقدم البشري والحضارة الإنسانية.

وربما كان لفشل جهود التحديث والنهوض على الأسس القومية والعلمانية والتغريبة في العالم الإسلامي طيلة الثلث الثاني من القرن العشرين، بعض الأثر في إظهار الحاجة إلى البديل الإسلامي وإثاعة شعاراته في الأسلمة والتحول الإسلامي. وربما تأثر خطاب الصحوة الإسلامية مع بداية الثلث الأخير من القرن بذلك. وقد يكون الضغط الشعبي للجماهير المسلمة ودعمها لمطالب الحركات الإسلامية قد جعل الأنظمة الحاكمة في بعض الأقطار الإسلامية تتبنى بعض الشعارات الإسلامية وتتخذ بعض الإجراءات العملية في التحول الإسلامي. وبعيداً عن تفسير النوايا فقد جاءت بعض الإجراءات منفّرة وقاصرة وأسهمت في تشويه شعارات الأسلمة. كذلك فقد شعرت بعض الأنظمة الحاكمة في أغا لا تستطيع تحمل الضغوط التي تمارسها قوى الميمنة العالمية لمواجهة جهود التحول الإسلامي وإحباط 
تياراته السياسية، وأها أيضاً لا تستطيع أن تستغني عن دعم الكوادر والخبرات المحلية التي نشأت وتكونت في المناخ العلماني التغربي، فكانت لها اعتراضاتما وتحفظاتها حول الدعوة إلى الأسلمة والتعاون مع دعاتما.

وفي المقابل أدت المعاناة القاسية التي واجهتها الحركات الإسلامية في صراعها مع أنظمة الحكم إلى استبعاد العنف والتطرف وسيلة في تحقيق أهدافها في الأسلمة، ووجدت في الحريات المحدودة المتاحة مناخاً لحشد الضغط الشعبي حول شعاراةها، ووجدت نفسها أيضاً في بعض الحالات في مواقف تفاوضية مع خصومها الذين يرفعون شعارات مختلفة، ومع جماهيرها التي تريد تحقيق مصالح دنيوية ونتائج عملية.

ويبين الرصد الدقيق والتحليل العميق وإعمال النظرة الكلية أن وضع شعار الأسلمة موضع التطبيق في جهود التحول الإسلامي في عدد من المجتمعات، يحتاج إلى توفير متطلبات أكثر بكثير مما كان يظن كثير من الناس. فالقضية ليست تشريعاً فوقياً يفتي بالحِل لما كان ممنوعاً وبالحرمة لما كان مباحاً، بل تبين أن ثمة حاجة لدراسة جدوى البدائل وتقدير تبعاتا، مع الأخذ بعين الاعتبار ضرورات الترفق والتدرج في تربية الاحساسات الشعبية والأذواق الاجتماعية وهيئها للبديل الإسلامي. ولا شك في أن ذلك يحتاج إلى فهم القضايا المعاصرة في مسائل السياسة والاقتصاد والاجتماع وسنن التحول النفسي والتغير الاجتماعي، وفي ضوء نصوص الإسلام ومقاصده مع استيعاب كامل لمعطيات المعرفة الإنسانية والتجربة البشرية.

الحاجة إلى الفهم والاستيعاب إذن هي التي تدعو إلى بناء أساس معرفي قوي لمواقفنا في الحياة وسلوكنا بحاه قضاياها، والمنهجية الإسلامية هي ذلك الإطار الاعتقادي والقيمي الذي يحكم نظامنا المعريف. ومن هنا كانت إسلامية المعرفة. 